\title{
Erratum to: The thermal stability of radiation-induced defects in illite
}

\author{
T. Riegler ${ }^{1} \cdot$ T. Allard ${ }^{2} \cdot$ D. Beaufort ${ }^{1} \cdot$ J.-L. Cantin ${ }^{3} \cdot$ H. J. von Bardeleben ${ }^{3}$
}

Published online: 10 December 2015

(C) Springer-Verlag Berlin Heidelberg 2015

\section{Erratum to: Phys Chem Minerals \\ DOI 10.1007/s00269-015-0770-9}

In the original publication, the last author's name was incorrect. The correct name of the last author should be H. J. von Bardeleben.

The original article has been updated accordingly.

The online version of the original article can be found under doi:10.1007/s00269-015-0770-9.

T. Riegler

thomas.riegler@univ-poitiers.fr

T. Allard

Thierry.allard@impmc.umpmc.fr

D. Beaufort

daniel.beaufort@univ-poitiers.fr

J.-L. Cantin

Jean-louis.cantin@insp.jussieu.fr

H. J. von Bardeleben

jurgen.vonbardeleben@insp.jussieu.fr

1 IC2MP, UMR 7285, Université de Poitiers, Bât. B35, 6 rue Michel Brunet, TSA 51106, 86073 Poitiers Cedex 9, France

2 IMPMC, UMR 7590, Institut de Recherche pour le Développement, Muséum d'Histoire Naturelle, Université Pierre et Marie Curie, 4 Place Jussieu, 75252 Paris Cedex 05,

France

3 INSP, Campus Jussieu, 75252 Paris Cedex 05, France 